\title{
Design optimization of Cassegrain telescope for remote explosive trace detection.
}

\author{
BHAVSAR, K., ESELLER, K.E., PRABHU, R.
}




\title{
Design Optimization of Cassegrain Telescope for Remote Explosive Trace Detection
}

\author{
Kaushalkumar Bhavsar*a, K E Eseller ${ }^{\mathrm{b}}$, Radhakrishna Prabhu ${ }^{\mathrm{a}}$ \\ ${ }^{a}$ School of Engineering, Robert Gordon University, Aberdeen, United Kingdom; ${ }^{b}$ Department of \\ Electrical \& Electronics Engineering, Atilim University, Ankara, Turkey
}

\begin{abstract}
The past three years have seen a global increase in explosive-based terror attacks. The widespread use of improvised explosives and anti-personnel landmines have caused thousands of civilian casualties across the world. Current scenario of globalized civilization threat from terror drives the need to improve the performance and capabilities of stand-off explosive trace detection devices to be able to anticipate the threat from a safe distance to prevent explosions and save human lives.

In recent years, laser-induced breakdown spectroscopy (LIBS) is an emerging approach for material or elemental investigations. All the principle elements on the surface are detectable in a single measurement using LIBS and hence, a stand-off LIBS based method has been used to remotely detect explosive traces from several to tens of metres distance. The most important component of LIBS based standoff explosive trace detection system is the telescope which enables remote identification of chemical constituents of the explosives. However, in a compact LIBS system where Cassegrain telescope serves the purpose of laser beam delivery and light collection, need a design optimization of the telescope system. This paper reports design optimization of a Cassegrain telescope to detect explosives remotely for LIBS system. A design optimization of Schmidt corrector plate was carried out for Nd:YAG laser. Effect of different design parameters was investigated to eliminate spherical aberration in the system. Effect of different laser wavelengths on the Schmidt corrector design was also investigated for the standoff LIBS system.
\end{abstract}

Keywords: LIBS, Spectroscopy, Schmidt-Cassegrain, Aberrations.

\section{INTRODUCTION}

In recent years, acts of terrors and warfare threats are increasing globally posing a severe threat to humanity. Use of explosives, anti-personnel landmines and chemical warfare agents in such terror activities are amongst the major concern, as illustrated by recent terror attacks in Manchester (UK) and Boston (marathon bombing) and several terror attacks on civilians in the Middle East. Advancement in technology has led to improvised terror weapons, especially the explosive devices and are commonly used by insurgents and terrorists to cause military and civilian casualties as well as inflict severe damage to infrastructures. Worldwide, government agencies are tasked to identify the evolving terror threats to national security such as improvised explosive devices (IEDs), concealed explosives, airborne chemical and biological materials without putting the people in harm. On the other hand, even after years of ending wars, unexploded explosives and landmines and other weapons are a threat to civilians ${ }^{1}$. To combat the rise in such terror activities globally, there is a need to improve the performance of explosive detection devices and their capabilities.

Detection of explosives is a major challenge which is continuously being addressed as the new explosive materials evolve. There are many methods to detect such explosive materials, however, most requires samples to be transported to the instrument or in a close proximity to the instrument for the analysis and therefore pose a threat. Standoff detection methods allow the remote detection of the explosive materials from a safe distance and reduce the potential of severe damage and harm. Some methods allow detection of suspicious bulk objects while others detect trace explosive materials, their precursors and breakdown products. The challenges in standoff detection is to maximize the screening distance while reducing the effect of background and interference signals from various environmental parameters to enhance the sensitivity.

Many standoff detection techniques are based on optical spectroscopy where the signal from the specimen is correlated to database of wavelength or wavenumber spectrum of specific chemical compounds used in explosives. Such optical 
methods used for trace detection requires a certain level of energy of specific wavelengths related to explosive materials in a backscattered light from the target ${ }^{2-5}$. One such technique is Laser Induced Breakdown Spectroscopy (LIBS). LIBS is a spectroscopy based analytical technique which uses the light (plume) emitted by the sample upon laser irradiation to determine its elemental composition. This technique provides real-time, rapid, in situ multi-elemental analysis with a minimal or no sample preparation. LIBS allow detection of all the principle elements on the target surface in a single measurement. The technique has been widely used in chemical and molecular analysis of bulk materials (i.e. solid, liquid and gas) to detect trace amount of concentration in a range of ppm and holds a promise of high performance for explosive material detection ${ }^{6,7}$. Determination and identification of explosive materials such as 1,3,5-trinitroperhydro-1,3,5-triazine (RDX), 2,4,6-trinitrotoluene (TNT), octahydro-1,3,5,7-tetranitro-1,3,5,7-tetrazocine (HMX), pentaerythritol tetranitrate (PETN), and propellant materials such as JA2 and M43 have been reported using LIBS ${ }^{3,8}$. Atomic emission lines of carbon, oxygen, nitrogen and hydrogen which span across the UV to NIR spectral region are commonly used to identify these materials $^{3,6}$.

Typically, a high-power laser is used to irradiate the surface of the target with an ultra-short pulse of energy to generate the plume/plasma. The generated plume (light spectrum) is collected by optical telescope and is transferred to the spectrometer for the analysis ${ }^{2}$. Therefore, the performance and standoff distance of the system directly depends on the telescope design. Efficient telescope design is required to improve the sensitivity of the LIBS.

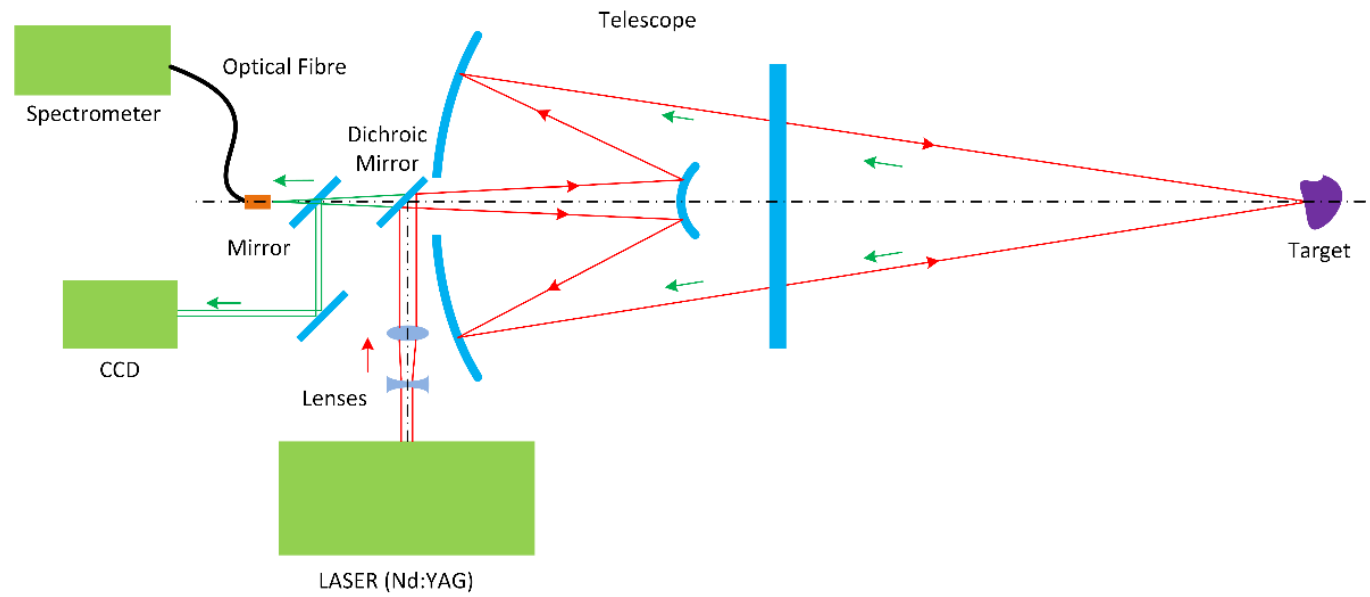

Figure 1. Experimental setup of LIBS with Schmidt-Cassegrain telescope for standoff detection of explosives.

A very basic telescope design is the Newtonian telescope. It consists of parabolic primary mirror and a flat secondary mirror at the axial focal point. The flat diagonal mirror reflects the light to the side of the tube. However, this telescope mainly suffers from comma and off-axis aberrations due to the optical design. Furthermore, a secondary flat mirror and its supporting structure within the tube obstruct the incoming light. Newtonian telescope consists of open design and therefore suffers from dust and highly affected by other environmental conditions in the field ${ }^{9,10}$. To overcome the issues with the Newtonian telescope design, modified design was introduced by Guillaume Cassegrain in which he replaced the diagonal flat mirror with the convex secondary mirror. The secondary spherical mirror converge the light beam and directs it through a hole in the primary to a new focus. The secondary spherical mirror not only folds the light path but also increases the effective focal ratio of the primary mirror. This results in using a longer focal length to be compressed in a very short tube and provide high magnification and enable compact telescope design suitable for the standoff applications ${ }^{9,11}$.

Cassegrain telescopes often referred as the catadioptric telescopes, offers versatility and high magnifying power with a compact design. The design consists of spherical primary and secondary mirror which is easy to manufacture. However, the Cassegrain telescope suffers from spherical aberrations due to the geometrical properties of the spherical surfaces used in the design ${ }^{9-11}$. Bernhard Schmidt eliminated the spherical aberration of the mirror by inserting a corrector plate. A simplest design of Schmidt-Cassegrain can be achieved by incorporating the full-aperture corrector for the spherical aberration and a single concave mirror ${ }^{12}$. The design concept is based on the unique property of spherical mirror with aperture stop at the center of curvature. A Schmidt corrector plate is placed at the front of the tube and it seals the telescope tube. Hence, the design itself guard against the dust and environmental currents. Therefore, Schmidt-Cassegrain design was used in many standoff optical sensing applications. Schmidt-Cassegrain telescope design was used in the standoff 
LIBS based detection to collect the signal from the object ${ }^{13}$. Standoff detection of explosive particles using Raman spectroscopy was reported by Ostmark et al. ${ }^{4}$ using Schmidt-Cassegrain telescope. Furthermore, Glimtoft et al. ${ }^{5}$ have also used the same design to detect explosive particles using Raman spectroscopy. However, in all these applications, laser beam was focused through separate optics. To utilize the same telescope system for the laser beam delivery requires the design optimization. This paper reports the design optimization of a Cassegrain telescope for a standoff LIBS system to detect explosives. A widely used Schmidt-Cassegrain telescope configuration for the above applications was investigated. Design of a Schmidt corrector plate for the Nd:YAG Laser was carried out and various design parameters were discussed to optimize the design for the specific laser wavelength. Effect of spherical aberrations on the system was investigated. Effect of different Nd:YAG Laser wavelengths $(266 \mathrm{~nm}, 532 \mathrm{~nm}, 1064 \mathrm{~nm})$ on the Schmidt corrector design was studied.

\section{THEORY}

\subsection{Cassegrain telescope design}

Cassegrain telescope is two mirror system which consists of a concave primary mirror and a convex secondary mirror ${ }^{10}$. Light coming from the object is reflected by primary mirror towards secondary mirror. After the second reflection from secondary mirror, light focuses on the point located on the vertex usually behind the primary mirror through a hole in the center of the primary. The typical design configuration of Cassegrain telescope is shown in the Figure 2.

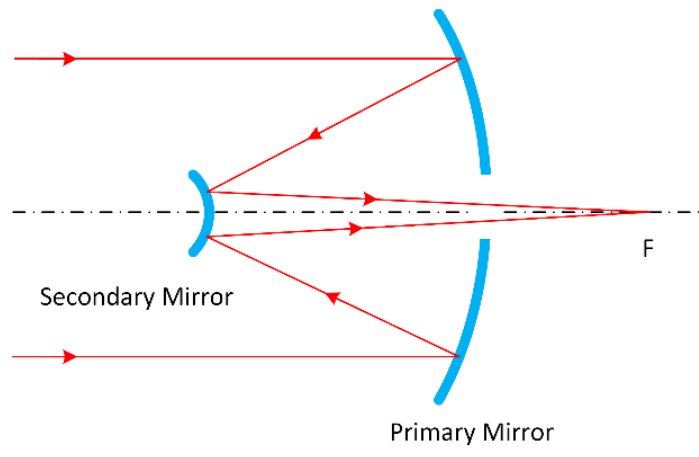

Figure 2. Schematic of Cassegrain telescope design/configuration.

Symmetry of spherical geometry attributes to the optical path difference in the incoming rays coming from the object at infinity leading to aberration called spherical aberration. Figure 3(a) illustrates the longitudinal spherical aberration of primary spherical mirror. Rays reflected by the spherical mirror do not meet at the same point on the vertex. The rays near the edges of the mirror (marginal rays) crosses the vertex very close to the mirror while paraxial rays closer to the center point of the mirror crosses the vertex at a more distant point. The deviation of the wavefront is minimum at the best focus point between the paraxial and marginal focal points. Spherical aberration affects the area across the image, including the very center. Therefore, it is very important to correct it than that of other inherent conic surface aberrations, which affect the outer area of the image.

Aberration coefficient of the spherical mirror surface, $s$, can be determined by the expression ${ }^{14}$ :

$$
S=\frac{1+K}{4 R^{3}}
$$

where $K$ is the conic constant, $R$ is the radius of curvature of the mirror and wavefront error can be expressed as ${ }^{14}$ :

$$
W_{s}=s d^{4}
$$

where $d$ is the radius of aperture (mirror). Figure 3(b) shows the calculated spherical aberration diagram for the Cassegrain telescope system. The red and blue color indicates the spherical aberration due to rays arriving at different focal point across the image surface which leads to blur in the image area. This spherical aberration of the system can be corrected by addition of Schmidt corrector plate in the system. 



Figure 3 (a) shows the spherical aberration from the primary mirror and (b) calculated spherical aberration diagram of the Cassegrain telescope.

\subsection{Schmidt-Cassegrain corrector plate}

The Schmidt-Cassegrain telescope design consists of a thin aspheric Schmidt corrector plate to compensate for the spherical mirror aberrations of the Cassegrain system. The Schmidt corrector plate $\mathrm{P}$ is placed at the center of curvature of the primary mirror as shown in Figure 4(a). The purpose of the Schmidt corrector plate is to modify the path length of the incoming rays so that all the rays come to a focus at single point after reflection from the mirror. Therefore, the Schmidt corrector plate compensates for the optical path length difference created by the spherical mirror surface and its profile has to be inverse of the aberrated wavefront. Schmidt corrector plate design is shown in Figure 4(b). The Schmidt corrector consists of two surfaces: one flat surface and second aspheric surface. The shape of the second surface, i.e. thickness, aperture, height, radius of curvature, position, varies to correct the spherical aberration of the system.

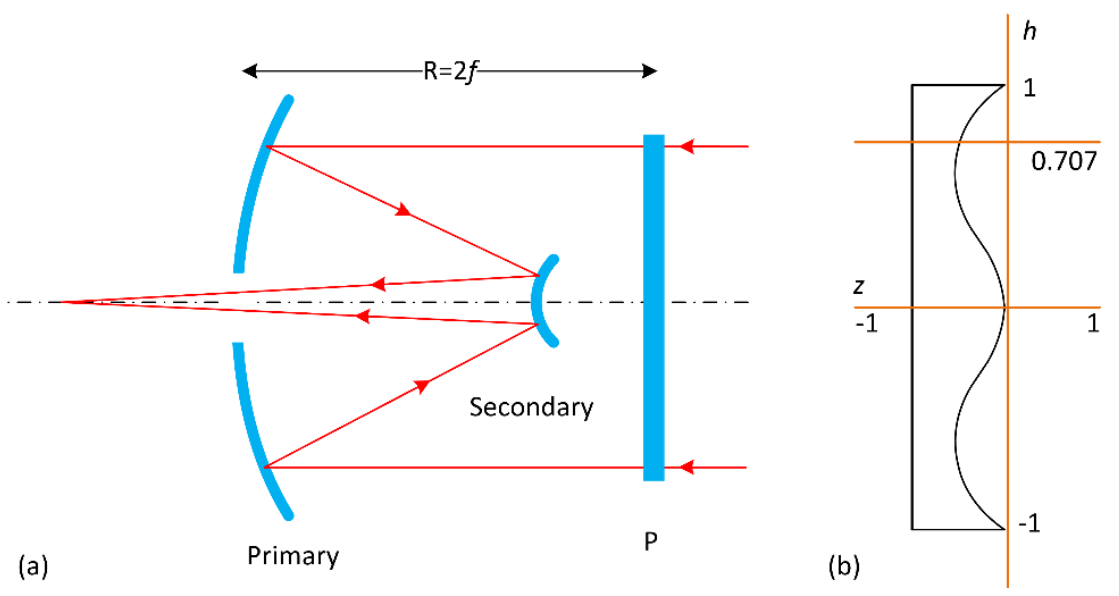

Figure 4 (a) Schematic of Schmidt-Cassegrain telescope configuration and (b) Schmidt corrector plate design for the Cassegrain telescope.

The depth profile of the Schmidt corrector plate need to be calculated to correct the spherical aberration. This adds the path length to compensate the optical path in effect to bring all the reflected rays to paraxial focus. The depth $z$ of the corrector surface can be calculated using the following expression ${ }^{15}$ :

$$
z=\frac{\left(\rho^{4}-\Lambda \rho^{2}\right) d^{4}}{4(1-n) R^{3}}
$$

where $\Lambda$ is the relative focus parameter, $d$ is the aperature radius, $\rho$ is the normalized height in the pupil $\rho=h / d, n$ is the refractive index of glass and $R$ is the radius of mirror curvature. Neutral zone location of the Schmidt corrector plate is determined by the corrector's focus parameter $\Lambda$, while its depth varies with the aperature radius $d$.

The wavefront errors for the lower-order (third-order) spherical aberration at the best focus point for the SchmidtCassegrain telescope can be given by ${ }^{15}$ : 


$$
W S=\frac{\left(s_{c r}+s_{M}\right) d^{4}}{4}
$$

where $s_{c r}$ is the spherical aberration coefficient of the corrector, $s_{M}$ is the sum of individual mirror spherical aberration coefficients, $s_{M}=s_{1}+s_{2}$.

$$
S_{1}=\frac{1+K_{1}}{4 R_{1}^{3}} \text { and } S_{2}=-\frac{k\left[K_{2}+\left(\frac{m+1}{m-1}\right)^{2}\right]\left(1-\frac{1}{m}\right)^{3}}{4 R_{1}^{3}}
$$

where $m$ is the secondary magnification ratio and $k$ is the relative ray height at the secondary.

To make the spherical aberration of the system to zero, the spherical aberration coefficient of the corrector $S_{c r}$ must be equal and opposite to the sum of the spherical aberration coefficients of mirrors $S_{M}$. The spherical aberration coefficient $S_{c r}$ depends on the corrector power $P$ given by $P=1-k(m-1)(m+1)^{2} / m^{3}$. Therefore, the system wavefront error at the best focus point can be expressed as:

$$
W_{S}=\frac{\left\{P-\left(K_{1}+1\right)-k\left[K_{2}+\left(\frac{m+1}{m-1}\right)^{2}\right]\left(1-\frac{1}{m}\right)^{3}\right\} d^{4}}{64 R_{1}^{3}}
$$

where $P$ is the needed corrector power to make the spherical aberration of the system to zero.

\section{RESULTS AND DISCUSSIONS}

A standoff LIBS system consists of Nd:YAG laser, Schmidt-Cassegrain telescope and a high-resolution broadband spectrometer. An 8-inch Schmidt-Cassegrain telescope was designed for the standoff LIBS system for trace detection of explosives. The diameter of the primary mirror and the focal length were $200 \mathrm{~mm}$ and $2000 \mathrm{~mm}(8$-inch $f / 10$ telescope design). The relative depth profile of the Schmidt corrector plate was calculated for different longitudinal aberration $L$ normalized to $2(0 \leq \Lambda \leq 2)$ as shown in Figure 5. The focus parameter $\Lambda$ was considered zero at paraxial focus point and two at marginal focus point. Best focus of the system and the location of least confusion circle were at $\Lambda=1$ and 1.5 , respectively. The Nd:YAG laser of wavelength $1064 \mathrm{~nm}$ was used for the design calculation. A BK7 glass was used for the Schmidt corrector plate design.

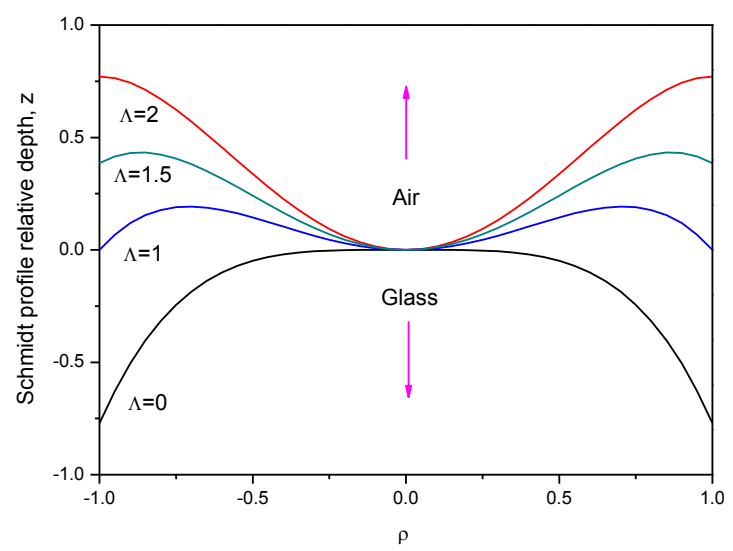

Figure 5 shows the Schmidt corrector relative depth profile with normalized pupil height for different focus parameter.

For a $200 \mathrm{~mm} f / 2 / 10$ Schmidt-Cassegrain telescope with primary magnification of 2 , gives the secondary magnification $m$ $=5$. Both mirrors used in the design are spherical, therefore conic constant $K_{1}$ and $K_{2}$ of the primary and secondary mirrors are zero. Radius of curvature of the primary mirror $R_{1}=4000 \mathrm{~mm}$. Hence, the spherical aberration coefficients, $s_{1}$ and $s_{2}$ are $3.9063 \mathrm{e}-12$ and $1.1250 \mathrm{e}-12$ respectively (eq. 5). To make this spherical aberration free system, the needed corrector power $P$ is 0.7120 .

Furthermore, the effect of laser wavelength on the Schmidt corrector design was studied. The Nd:YAG laser is the most commonly used laser with fundamental wavelength of $1064 \mathrm{~nm}$ for LIBS application. Second and fourth harmonic wavelengths $\left(532 \mathrm{~nm}\right.$ and $266 \mathrm{~nm}$ ) of the Nd:YAG laser are also common for a wide range of LIBS application ${ }^{2,6}$ Therefore, it is important to study the effect of these commonly used laser wavelengths in the design of Schmidt corrector 
plate. Figure 6 shows the variation in the Schmidt corrector relative depth profile at the best focus location for different $\mathrm{Nd}$ :YAG laser wavelengths. It was observed that relative depth of the Schmidt corrector changes with wavelength with maximum deviation at 0.707 . The observed decrease in the corrector thickness at $\rho=0.707$ was $2 \%$ and $8 \%$ for the 532 and $266 \mathrm{~nm}$ wavelengths with reference to fundamental wavelength $(1064 \mathrm{~nm})$. Furthermore, the deviation in relative depth doubles at $\rho=0.707$ going from best focus point $(\Lambda=1)$ to the location of least confusion circle $(\Lambda=1.5)$.

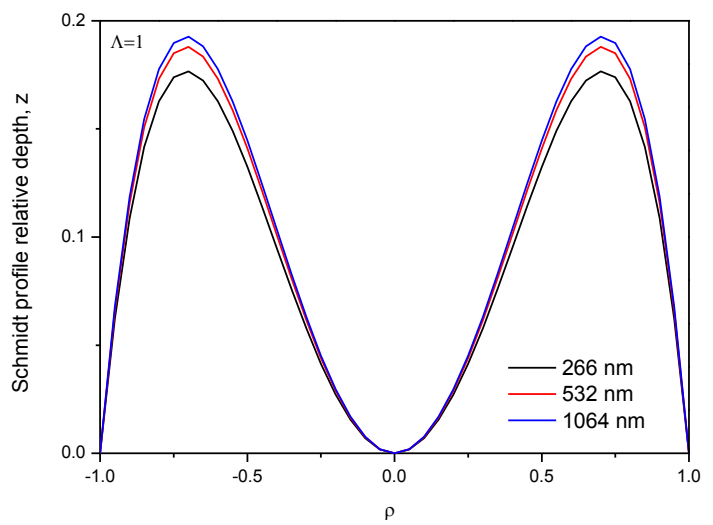

Figure 6 shows the Schmidt corrector plate relative depth profile for different laser wavelengths.

Obtained results suggests that addition of Schmidt corrector plate in the Cassegrain telescope system to correct the spherical aberration introduces the spherochromatism in the system. The optimally designed corrector plate for one wavelength overcorrects the smaller wavelengths and undercorrects the higher wavelengths with reference to the designed wavelength. Furthermore, addition of the Schmidt corrector in the ray path introduces small refraction angle and therefore, the ray is being directed at slightly different position on the primary mirror which results into slightly different magnification of the system. Overall, it affects the focusing of the laser beam and its divergence which needs to be investigated.

\section{CONCLUSION}

A standoff LIBS system with Schmidt-Cassegrain telescope is proposed to deliver the Laser beam and collect the signal from the trace explosives. Design optimization of the Schmidt corrector plate was investigated for the LIBS system using $\mathrm{Nd}$ :YAG laser. Optimization of Schmidt corrector depth profile was discussed for the Nd:YAG laser of $1064 \mathrm{~nm}$ wavelength at different focus parameters to correct the spherical aberration of the 8-inch f/10 Cassegrain telescope. Spherical aberration of the Cassegrain telescope was calculated and required corrector power was identified to make the spherical aberration free system. Addition of the Schmidt corrector plate in the Cassegrain telescope system to correct the spherical aberration introduces the spherochromatism in the system. Calculated results for different laser wavelengths suggests that the Schmidt corrector should be optimized for the specific wavelength to avoid the spherochromatism. Further work will be carried out considering all the aberrations in the Schmidt-Cassegrain telescope and their effects on the LIBS system.

\section{REFERENCES}

[1] Mousavi, B., Soroush, M. R., Masoumi, M., Khateri, S., Modirian, E., Shokoohi, H., Fatemi, M. J., Hematti, M. A., Soroush, M., Ghassemi-Broumand, M., Rassafiani, M., Allami, M., Nouri, F., Yavari, A., Ganjparvar, Z., Kamyab, M. and Mirsadeghi, S. A., "Epidemiological Study of Child Casualties of Landmines and Unexploded Ordnances: A National Study from Iran," Prehosp.Disaster Med. 30(5), 472-477 (2015).

[2] Radziemski, L. J. and Cremers, D. A., [Handbook of Laser Induced Breakdown Spectroscopy], Wiley, West Sussex, UK, 432 (2013).

[3] De Lucia, F., Harmon, R., McNesby, K., Winkel, R. and Miziolek, A., "Laser-induced breakdown spectroscopy analysis of energetic materials," Appl.Opt. 42(30), 6148-6152 (2003).

[4] Ostmark, H., Nordberg, M. and Carlsson, T. E., "Stand-off detection of explosives particles by multispectral imaging Raman spectroscopy," Appl.Opt. 50(28), 5592-5599 (2011). 
[5] Glimtoft, M., Baath, P., Saari, H., Makynen, J., Nasila, A. and Ostmark, H., "Towards eye-safe standoff Raman imaging systems," Detection and Sensing of Mines, Explosive Objects, and Obscured Targets Xix 9072, UNSP 907210 (2014).

[6] Gottfried, J. L., De Lucia, F. C. Jr., Munson, C. A. and Miziolek, A. W., "Laser-induced breakdown spectroscopy for detection of explosives residues: a review of recent advances, challenges, and future prospects," Analytical and Bioanalytical Chemistry 395(2), 283-300 (2009).

[7] DeLucia, F., Samuels, A., Harmon, R., Walters, R., McNesby, K., LaPointe, A., Winkel, R. and Miziolek, A., "Laser-induced breakdown spectroscopy (LIBS): A promising versatile chemical sensor technology for hazardous material detection," Ieee Sensors Journal 5(4), 681-689 (2005).

[8] Gottfried, J. L., De Lucia, F. C. Jr., Munson, C. A. and Miziolek, A. W., "Strategies for residue explosives detection using laser-induced breakdown spectroscopy," J.Anal.At.Spectrom. 23(2), 205-216 (2008).

[9] Wilson, R., [Reflecting Telescope Optics I], Springer, Berlin Heidelberg, 545 (1996).

[10] Romano, A. and Cavaliere, R., 'Newtonian and Cassegrain Telescopes', [Geometric Optics: Theory and Design of Astronomical Optical Systems Using Mathematica $\left.{ }^{\circledR}\right]$, Springer International Publishing, Cham, 107-118 (2016).

[11] Mullaney, J., 'Reflecting Telescopes', [A Buyer's and User's Guide to Astronomical Telescopes \& Binoculars], Springer London, London, 35-45 (2007).

[12] Weisheit, B., 'Optics and Telescopes', [Handbook of Practical Astronomy], Springer Berlin Heidelberg, Berlin, Heidelberg, 41-93 (2009).

[13] Gong, Y., Choi, D., Han, B., Yoo, J., Han, S. and Lee, Y., "Remote quantitative analysis of cerium through a shielding window by stand-off laser-induced breakdown spectroscopy," J.Nucl.Mater. 453(1-3), 8-15 (2014).

[14] Mahajan, V. N., 'Calculation of Primary Aberrations: Reflecting and Catadioptric Systems', [Optical Imaging and Aberrations: Part I. Ray Geometrical Optics], SPIE, Bellingham, Washington, 365-436 (1998).

[15] Schroeder, D. J., 'Schmidt Telescopes and Cameras', [Astronomical Optics], Academic Press, San Diego, 164184 (2000). 